\title{
Matrix manipulation and interpretation of data related to AIDS transmission
}

\author{
MICHAEL YOST and FREDERICK J. BREMNER \\ Trinity University, San Antonio, Texas
}

This research describes how a currently available spreadsheet program can be set up to analyze sociographic data. An algorithm developed by Jamrich (1960) is presented, along with both the mathematical and the spreadsheet equations. An example of the application of the algorithm and equations to AIDS transmission data is presented.

Ever since Moreno (1934) introduced sociometry to the behavioral sciences, it has been utilized in many types of investigations. In Moreno's original conception, sociometric choices were represented by a sociograph. In this representation, a circle usually indicated an individual and arrows connecting individuals indicated the choices that were made. Arrows pointing away from a subject, toward another individual, usually meant that this individual was chosen by the subject. By using appropriate symbols, we may indicate that $A$ chooses $B$, rejects $B$, or ignores $B$. The problem with sociographs was that they were not unique representations of the data. A sociograph may be drawn in many ways, and, when a number of relationships are considered, we may obtain a number of crossing lines that confound interpretation.

One of the areas of refinement in sociometry has been the development of techniques for the matrix representation of sociometric data. A matrix is well suited for this purpose. The matrix can be arranged with the subjects listed in the same order, both down the side and across the top. If we designate the rows as the choices made by the subjects, we may represent each sociometric choice made by an individual by putting a " 1 " in the column associated with the person he/she chooses; otherwise, we leave the cell blank. If we sum down the columns, we have the number of sociometric choices received by the individual. Unlike the sociograph, this representation of the data is unique. Many elaborate schemes for the utilization of sociometric data have been derived (Beum \& Brundage, 1950; Glanzer \& Glaser, 1959). Some of them, like Beum and Brundage's procedure, depend on the hierarchical rearrangement of a matrix. Another approach has involved raising matrices to some power. This technique was introduced by Festinger (1949) and elaborated on by Luce and Perry (1949) and by Luce (1950). A modification of the Festinger procedure has been suggested by Jamrich (1960).

Correspondence should be addressed to Michael Yost, Office of the President, Trinity University, 715 Stadium Drive, San Antonio, TX 78212 .
Jamrich reasoned that in a squared matrix we have second-order relationships, which can be interpreted as second-order communications. In other words, Tom contacts Bob through an intermediary whom we might call Sam. Jamrich argued that in sociometric situations, if we utilize the squared matrix and then add back the original matrix to it, we will have a matrix that weights the choices by the status of the chooser. If the subject receives a nomination from someone who is highly chosen, the sociological implications of this choice are probably different from the implications associated with a nomination from a less frequently chosen person. The Jamrich procedure weights the sociometric choice so that it reflects differences in the status of the chooser.

\section{Matrix Manipulation}

An understanding of basic matrix manipulation is required if the reader is to follow the ensuing discussion. Although a simple $2 \times 2$ matrix is seldom encountered in sociometric data, it may be used here for illustrative purposes. Suppose that there are two $2 \times 2$ matrices, $A$ and $B$. Both matrices have two rows and two columns. These two matrices and their elements can be represented by

$$
A=\left(\begin{array}{ll}
a_{11} & a_{12} \\
a_{21} & a_{22}
\end{array}\right) \text { and } B=\left(\begin{array}{ll}
b_{11} & b_{12} \\
b_{21} & b_{22}
\end{array}\right) \text {. }
$$

The first (left) of the two subscripts represents the row, and the second (right) represents the column.

The addition of these two matrices results in a third matrix with an equal number of rows and columns. The addition process can be represented by

$$
A+B=\left[\begin{array}{ll}
\left(a_{11}+b_{11}\right) & \left(a_{12}+b_{12}\right) \\
\left(a_{21}+b_{21}\right) & \left(a_{22}+b_{22}\right)
\end{array}\right] .
$$

The multiplication of two matrices is somewhat more complex. Basically, the elements in the first row of the first matrix are multiplied by the elements in the first column in the second matrix, summed, and then placed as the top left element in the product matrix. This process is then completed with each of the rows and columns. 
The format for the process is as follows:

$A \times B=\left[\begin{array}{ll}\left(a_{11} \cdot b_{11}+a_{12} \cdot b_{21}\right) & \left(a_{11} \cdot b_{12}+a_{12} \cdot b_{22}\right) \\ \left(a_{21} \cdot b_{11}+a_{22} \cdot b_{21}\right) & \left(a_{21} \cdot b_{12}+a_{22} \cdot b_{22}\right)\end{array}\right]$.

If we are presented with a matrix,

$$
M=\left(\begin{array}{lll}
0 & 1 & 1 \\
1 & 0 & 0 \\
1 & 1 & 0
\end{array}\right)
$$

then $M^{2}$ (which is equal to $M \times M$ ) would be

$$
M_{2}=\left(\begin{array}{lll}
0 & 1 & 1 \\
1 & 0 & 0 \\
1 & 1 & 0
\end{array}\right) \times\left(\begin{array}{lll}
0 & 1 & 1 \\
1 & 0 & 0 \\
1 & 1 & 0
\end{array}\right)=\left(\begin{array}{lll}
2 & 1 & 0 \\
0 & 1 & 1 \\
1 & 1 & 1
\end{array}\right),
$$

and $M+M^{2}$ would be

$$
M+M^{2}=\left(\begin{array}{lll}
0 & 1 & 1 \\
1 & 0 & 0 \\
1 & 1 & 0
\end{array}\right)+\left(\begin{array}{lll}
2 & 1 & 0 \\
0 & 1 & 1 \\
1 & 1 & 1
\end{array}\right)=\left(\begin{array}{lll}
2 & 2 & 1 \\
1 & 1 & 1 \\
2 & 2 & 1
\end{array}\right) .
$$

An example of the application of these matrix manipulations to a sociogram with a limited number of subjects follows.

Three subjects- $S_{1}, S_{2}$, and $S_{3}$-were each given an opportunity to make one or two choices of equal "strength." The selection matrix for the choices is

$$
\begin{aligned}
& S_{1} S_{2} S_{3}
\end{aligned}
$$

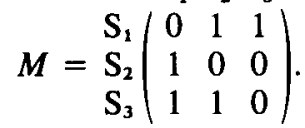

In this matrix, the values in the columns represent the number of times each subject was chosen by the (row) chooser. Both $S_{1}$ and $S_{2}$ were chosen twice, and $S_{3}$ was chosen once. The values in the rows of the matrix represent the choices made by each subject. Both $S_{1}$ and $S_{3}$ made two choices, and $S_{2}$ made only one choice. As is typical in the use of sociograms, none of the subjects chose themselves, and, as a result, the diagonal of the matrix contains zeros. The entries in this matrix represent the number of single or one-stage relations between the subjects. A traditional sociogram for this matrix would be

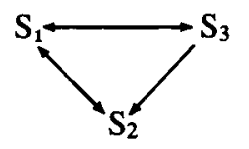

From the sociogram and $M$ (the choice matrix), we can see that there are five one-stage relations between the subjects. These include

$$
\begin{aligned}
& S_{1} \leftarrow S_{2} \\
& S_{1} \leftarrow S_{3} \\
& S_{2} \leftarrow S_{1} \\
& S_{2} \leftarrow S_{3} \\
& S_{3} \leftarrow S_{1}
\end{aligned}
$$

If we square the matrix $M$, we obtain

$$
\begin{aligned}
& \mathrm{S}_{1} \mathrm{~S}_{2} \mathrm{~S}_{3}
\end{aligned}
$$

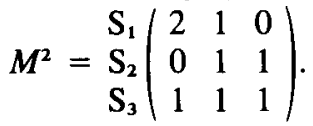

The off-diagonal entries in the rows and columns of this matrix indicate the number of two-stage relations between the subjects. From the entry at the intersection of the first (left) column and the last (bottom) row $\left(M_{31}\right)$, we can see that $S_{3}$ can communicate with $S_{1}$ through two stages,

$$
S_{1} \leftarrow S_{2} \leftarrow S_{3},
$$

whereas $S_{2}$ cannot communicate (there is no path) with $S_{1}$ in two stages. The elements on the diagonal of the matrix represent the number of mutual choices that were made. For example, $S_{1}$ chose both $S_{2}$ and $S_{3}$, and both $S_{2}$ and $S_{3}$ chose $S_{1} . S_{2}$ and $S_{3}$ each chose one other person who, in turn, chose them.

When $M$ and $M^{2}$ are added together, we obtain

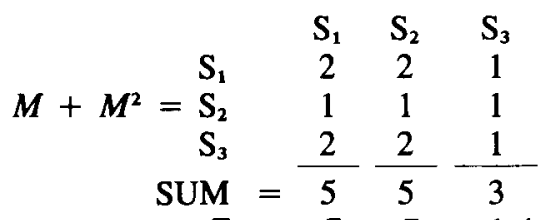

$$
\begin{aligned}
& Z=\begin{array}{lll}
7 & .7 & -1.4
\end{array}
\end{aligned}
$$

This new matrix represents the number of one- and twostage relationships between the subjects. The sum of the elements in each row indicates the number of "outward" one- and two-stage choice relationships made by the subjects. Similarly, the sum of the elements in the columns indicates the number of "inward" one- and two-stage chosen relationships made of the subjects. The "outward" relationships simply reflect the number and types of choices made; the "inward" relationships are an indicator of the "status" of each of the subjects. The "inward" relationships tend to be a more useful research tool than the "outward" ones. The column sums give the status of each of the subjects in the group. From the data presented here, $S_{1}$ and $S_{2}$ have equal status and $S_{3}$ has a lower status. The $Z$-score transformations of the column sums indicate the standard score status of each subject. The use of standard scores was suggested from research by Love, Hughes, and Yost (1969).

By using the column sums and the original choice matrix, we are able to identify subgroups or cliques and their relative status or strength. From their column sums, we can see that $S_{1}$ and $S_{2}$ have the most status and chose each other. Also, both $S_{1}$ and $S_{2}$ were chosen twice and $S_{3}$ was chosen only once. Therefore, $S_{1}$ and $S_{2}$ form a clique with a combined status of " 10 ." In situations that have larger numbers of subjects, there can be multiple cliques containing different numbers of members, with different levels of status.

\section{Calculating the Results}

Much of the research in the development of the sociogram had its origins in the 1930s and 1940s. Although 
much of the original work was relatively simplistic, some of the algorithms required the solution of several simultaneous equations, the squaring of matrices, and the cubing of matrices. In those early years, some of the calculations were done on an adding machine, but the majority of the calculations were done by hand. Throughout most of the $1950 \mathrm{~s}$ and $1960 \mathrm{~s}$, the calculations were done on mechanical adding machines. The effort expended on a two-selection, 20-person problem could be measured in person-days. One of the first computer solutions to these problems was published by Love et al. (1969). This program was written in FORTRAN; by today's standards, it was not "user friendly," and it required the use of a mainframe computer. It did, however, reduce the time needed to solve problems of reasonable sizes.

We have moved through a number of generations of computers; they have become more accessible, and they have become infinitely more user friendly. Also, there has been a proliferation of problem-solving software that does not require in-depth programming skills. One such program is Lotus 1-2-3, but there are other similar spreadsheet programs as well.

The development of a spreadsheet program to solve the research problems described earlier begins with the formatting of a screen onto which the data are to be entered. This data entry matrix is the same as the $M$ matrix. Developing this part of a spreadsheet requires the setting of column widths, numbers of rows, and columns, as well as the assigning of appropriate labels to them. Figure 1 gives a sample of a data entry page from a spreadsheet program. The only background equations or cell designators used on this page were those which enabled the names of the subjects entered in the column on the left of the data matrix to appear in the row at the top of the matrix.

Equations were also used to permit the names of the subjects to appear as row and column headings on the other matrices $\left(M^{2}\right.$ and $\left.M+M^{2}\right)$ of the entire spreadsheet program. Having the names of the subjects appear with the data and the solutions assists with the interpretation of the data.

The equations to square the data matrix are quite complex and lengthy. Suppose that there is a $10 \times 10$ data en-

DATA ENTRY MATRIX -

ENTER NAMES IN COLUMNS AND DATA IN THE MATRIX.

\begin{tabular}{|c|c|c|c|c|c|c|c|c|c|c|}
\hline & & & & & & RSON & 'CHOS & EN" & & \\
\hline P & & & & & & --- & $-\cdots$ & -- & & \\
\hline 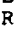 & & & S1 & & 53 & & S5 & & 57 & \\
\hline$S$ & NAME & & & \$2 & & S4 & & S6 & & 58 \\
\hline 0 & --- & & --- & -- & -- & -- & -- & - & -- & $\cdots$ \\
\hline $\mathbf{N}$ & & & & & & & & & & \\
\hline & S1 & --- & 0 & 1 & 0 & 1 & 0 & 0 & 0 & 0 \\
\hline C & s2 & --- & 1 & 0 & 0 & 0 & 0 & 0 & 0 & 1 \\
\hline H & S3 & $-\cdots$ & 0 & 0 & 0 & 1 & 0 & 0 & 1 & 0 \\
\hline 0 & 54 & $=--$ & 1 & 0 & 0 & 0 & 0 & 0 & 1 & 0 \\
\hline 0 & \$5 & --- & 0 & 0 & 0 & 0 & 0 & 1 & 0 & 1 \\
\hline $\mathbf{S}$ & 56 & ---- & 0 & 1 & 0 & 0 & 1 & 0 & 0 & 0 \\
\hline I & s7 & --- & 0 & 0 & 1 & 1 & 0 & 0 & 0 & 0 \\
\hline - & 58 & $-\cdots$ & 0 & 0 & 0 & 0 & 1 & 1 & 0 & 0 \\
\hline
\end{tabular}

Figure 1. Data entry matrix.

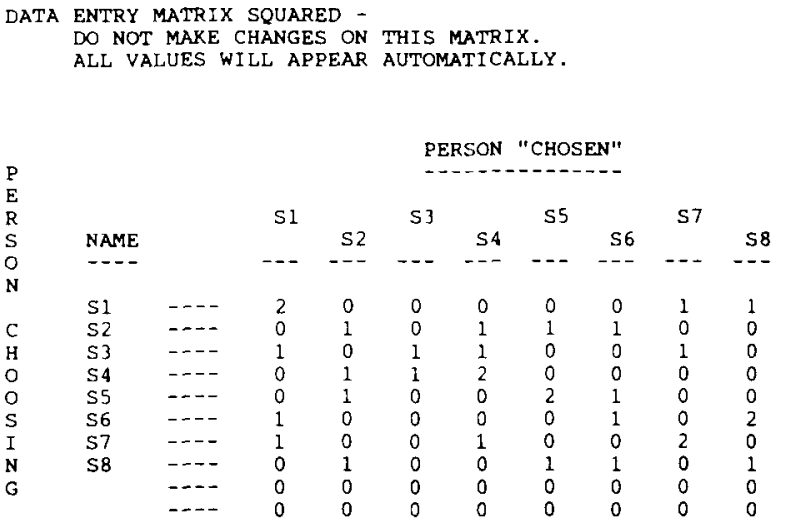

Figure 2. Data entry matrix squared.

try matrix. This would permit the solution of sociograms with up to 10 subjects. The data entry begins in cell D8 and extends to M17. The equation for the data element in the beginning of the first row and first column (D8) is

$$
(\mathrm{D} 8 * \mathrm{D} 8+\mathrm{E} 8 * \mathrm{D} 9+\mathrm{F} 8 * \mathrm{D} 10+\ldots \mathrm{M} 8 * \mathrm{D} 17) \text {. }
$$

This equation multiplies each element in the first row by the elements in the first column, sums them, and places the value in D8. There is a distinct advantage to writing this equation thus:

$$
\text { (\$D8*D8+\$E8*D9 +\$F8*D10+ . \$M8*D17). }
$$

This is the equation for the first data element of the first row. If the dollar sign (\$) notation is used in the equation, this equation can be copied into each of the other nine positions of the first row, and the relative positions of the equations for each column will be taken care of. The notation for the first data element in the second row is

$$
\text { (\$D9*D8+\$E9*D9+\$F9*D10+ . \$M9*D17). }
$$

Again, this equation may be copied across the entire row and the relative positions will be automatically adjusted. The format of the third-through-the-tenth rows follows the same progression as that of the first two rows.

There should never be a need to enter data in the $M^{2}$ matrix or to change any of the data elements listed on this figure. As the data are entered in the matrix on Figure 1, the calculations are automatically performed and the results placed on the matrix of Figure 2.

The equations needed to sum the $M$ and $M^{2}$ matrices are very simple. The sum matrix is the addition of the data element in one matrix, added to the data element in the same location in the other matrix. For example, if the first data element in the data matrix is in cell D8 and the same data element of the $M^{2}$ matrix is in cell D28, the equation for this position in the $M+M^{2}$ matrix is (D8 + D28). Because of the relative positioning of the data elements in the equations and the power of the spreadsheet program, only one equation needs to be written to develop the $M+M^{2}$ matrix. The initial equation can be copied to all $100(10 \times 10)$ cells of the spreadsheet, and the program will adjust for the relative row and column locations. 
The sum of the columns, the average column sum, and the standard deviations are calculated with the @SUM, @AVG, and @STD functions, respectively. The use of these functions requires little or no programming skills. The $Z$ scores were developed using a standard $Z$ score transformation which was translated into a spreadsheet format.

$$
\begin{aligned}
Z & =\frac{\text { Raw Score-Mean }}{\text { Standard Deviation }} \\
& =[(\text { Cell })-(@ \text { AVG })] / @ S T D
\end{aligned}
$$

The data that appear in Figures 1-3 were taken from research by Jamrich (1960). Although the data were from 8 subjects who were permitted to make up to two choices, it was analyzed with a matrix that would accommodate up to 10 subjects who were permitted to make any number of choices.

The three matrices can be set up in either of two formats. If your program permits only one two-dimensional spreadsheet to be in memory at one time, the three matrices can be set up in a continuous format in a single spreadsheet. Since this format is continuous, the user will be required to page between screens or use print output to do analyses. If your program permits the development and use of three-dimensional spreadsheets (or windowing), a page can be used for each of the three matrices. This permits the user to keep the data and the results on screen while working and analyzing data.

The interpretation of these data follows the same logic as presented earlier. Although the data presented initially were too simple to show the development of cliques, the data in Figures 1, 2, and 3 can be used to demonstrate the concept. From the column sums (Figure 3), we can see that $S_{4}$ and $S_{1}$ have the highest status of the group (8 and 7, respectively). From the diagonal of $M^{2}$ (Figure 2), we can see that $S_{4}$ has two mutual choices. From Figure 1 , we can see that these are $S_{1}$ and $S_{7}$. As a result, we can see that $S_{4}, S_{1}$, and $S_{7}$ form a clique with a total

DATA ENTRY MATRIX SQUARED + DATA MATRIX -
DO NOT MAKE CHANGES

\begin{tabular}{|c|c|c|c|c|c|c|c|c|c|c|}
\hline & & & & & & ERSON & "CHO & SEN" & & \\
\hline$E$ & & & & & & & & & & \\
\hline $\mathrm{R}$ & & & S1 & & S3 & & S5 & & s7 & \\
\hline $\mathbf{s}$ & NAME & & & 52 & & S4 & & S6 & & S8 \\
\hline 0 & --- & & -- & $-\cdots$ & -- & -- & -- & $-\cdots$ & -- & -- \\
\hline $\mathbf{N}$ & S1 & $\ldots$ & 2 & 1 & 0 & 1 & 0 & 0 & 1 & 1 \\
\hline C & $\mathrm{s} 2$ & --- & 1 & 1 & 0 & 1 & 1 & 1 & 0 & 1 \\
\hline H & s3 & $-\ldots$ & 1 & 0 & 1 & 2 & 0 & 0 & 2 & 0 \\
\hline 0 & S4 & -- & 1 & 1 & 1 & 2 & 0 & 0 & 1 & 0 \\
\hline 0 & S5 & $\ldots$ & 0 & 1 & 0 & 0 & 2 & 2 & 0 & 1 \\
\hline $\mathbf{S}$ & s6 & --- & 1 & 1 & 0 & 0 & 1 & 1 & 0 & 2 \\
\hline I & S7 & --- & 1 & 0 & 1 & 2 & 0 & 0 & 2 & 0 \\
\hline $\mathbf{N}$ & 58 & --- & 0 & 1 & 0 & 0 & 2 & 2 & 0 & 1 \\
\hline G & & --- & 0 & 0 & 0 & 0 & 0 & 0 & 0 & 0 \\
\hline & & --- & 0 & 0 & 0 & 0 & 0 & 0 & 0 & 0 \\
\hline & & & $-\cdots$ & -- & -- & - & --- & --- & - & --- \\
\hline & SUM & & 7 & 6 & 3 & 8 & 6 & 6 & 6 & 6 \\
\hline & z & & 0.8 & 0.0 & -2.3 & 1.5 & 0.0 & 0.0 & 0.0 & 0.0 \\
\hline & MEAN & & & & & & & & & \\
\hline & DEV & & 1.32 & & & & & & & \\
\hline
\end{tabular}

VALUES APPEAR AUTOMATICALLY.

Figure 3. Data entry matrix squared + data matrix. status of $21(8+7+6)$. Using similar logic, we can show that $S_{1}, S_{2}$, and $S_{4}$ form another clique with a status of $21(7+6+8)$. By virtue of their choices, Subjects 1,2 , 4 , and 7 form a subgroup of the original 8 subjects.

Suppose that the data in Figures 1, 2, and 3 were from 8 sexually intimate persons, and that the data in the $M$ matrix indicate the number of times that each of these 8 persons has "dated" in a particular period of time. The $M$ and the $M^{2}$ matrices show the number of one- and twostage relationships between these individuals, respectively. The $M+M^{2}$ matrix indicates the combined number of one- and two-step relationships, as well as the diagonal showing the number of mutual choices made by these individuals. The $Z$ scores represent the relative "popularity" of the 8 individuals within the group.

All of the individuals are not equally popular. $S_{1}$ and $S_{4}$ are the most popular, while subjects $S_{2}, S_{5}, S_{6}, S_{7}$, and $S_{8}$ are of average popularity, and $S_{3}$ is not very popular at all. From the number of mutual choices (the diagonal of the $M$ and $M+M^{2}$ matrices) and the number of singleand double-stage relationships, we can see that $S_{1}, S_{2}$, and $S_{4}$ form a clique, and that $S_{3}$ is largely excluded.

These matrix models can be used to illustrate concepts of contagion-such as the HIV virus-as it spreads from one individual to another. Suppose that sometime after these relationships were started that $S_{4}$, the individual with the highest status, tested positively for the HIV virus. Which of the individuals is in the greatest danger? From the three matrices, we can see that $S_{1}, S_{2}, S_{3}$, and $S_{7}$ all interacted directly or indirectly with $S_{4}$. While one person $\left(\mathrm{S}_{4}\right)$ tested positively, it is also possible that four other individuals could be in danger.

It is interesting to assume that $S_{3}$, the individual with the least status, tested positive to HIV and outline his patterns of interaction. $S_{3}$ interacted directly with $S_{4}$ and $S_{7}$. It can be seen from these data that $S_{3}$ has much less status and many fewer individuals are endangered. The status level of an individual within a group in which the health of one individual can endanger another determines the number of individuals endangered.

\section{Summary}

At the time of its development, a sociogram with its accompanying mathematics was a much more sophisticated research tool than the technology of its day would permit to be used with ease. In this respect, it shares a common heritage with a number of multivariate statistical techniques. With the development of mainframe computers, the technique became much easier to use. With the accessibility of microcomputers and the abundance of user-friendly software, the technique has been developed and used with ease by individuals who have minimal computer and programming skills.

From the researcher's point of view, the technique can now be used with groups of almost any size, with almost any number of choices. The time needed for analysis, availability of a program, or availability of a computer need no longer be used as an excuse for not making use of the technique. 
When run in a microcomputer, the technique can also be used as a tutorial tool. Since all of the calculations can be done almost instantly, many "what if" scenarios can be tested in a very short period of time: "What would happen to the status of __ subjects if a particular selection had or had not been made?" "What would happen if a particular subject had not been present?" "What would happen if there was another person who had made choices?" "What would have to happen to enhance the status of a particular subject?"

Tracing the transmission of HIV through the use of the mathematical approach is but one example of the application of an existing approach to a new problem. Moreover, this approach permits the easy identification of the individuals who may be involved in the transmission of the HIV virus.

One of the limits to the approach described here is that only primary and secondary stages of contact have been discussed. There is reason to believe that viruses such as HIV can be transmitted through three or more stages of interaction. The mathematics for these stages are readily available, and the computer programs exist which will permit investigators to work with data having this number of stages.

\section{REFERENCES}

Beum, C. E., Jr., Brundage, E. G. (1950). A method for analyzing the sociometric. Sociometry, 13, 141-145.

Festinger, L. (1949). The analysis of sociograms using matrix algebra. Human Relations, 2, 153-158.

Glanzer, M., \&laser, R. (1959). Techniques for the study of group structure and behavior: 1 . Analysis of structure. Psychological Bulletin, 56, 317-332.

JAMRICH, J. X. (1960). Application of matrices in the analysis of sociometric data. Journal of Experimental Education, 28, 249-252.

Love, W. A., Hughes, E. F., \& Yost, M. (1969). A program to compute the standard and the adjusted Jamrich sociometric indices. $B e$ havioral Science, 14, 82-83.

LUCE, R. D. (1950). Connectivity and generalized clique in sociometric group structure. Psychometrika, 15, 169-190.

LuCE, R. D., \& Perry, A. D. (1949). A method of matrix analysis of group structure. Psychometrika, 14, 95-116.

Moreno, J. L. (1934). Who shall survive? A new approach to the problem of human inter-relations. Washington, DC: Nervous \& Mental Disease Publishing Co. 